\title{
Enhancing Music Events Using Physiological Sensor Data
}

\author{
Thomas Röggla \\ Centrum Wiskunde \& Informatica \\ Science Park 123 \\ Amsterdam, The Netherlands \\ t.roggla@cwi.nl
}

\author{
Najereh Shirzadian \\ Centrum Wiskunde \& Informatica \\ Science Park 123 \\ Amsterdam, The Netherlands \\ najereh@cwi.nl
}

\author{
Zhiyuan Zheng \\ Centrum Wiskunde \& Informatica \\ Science Park 123 \\ Amsterdam, The Netherlands \\ z.zheng@cwi.nl
}

\author{
Alice Panza \\ Centrum Wiskunde \& Informatica \\ Science Park 123 \\ Amsterdam, The Netherlands \\ a.panza@cwi.nl
}

\begin{abstract}
This demo showcases a real-time visualisation displaying the level of engagement of a group of people attending a Jazz concert. Based on wearable sensor technology and machine learning principles, we present how this visualisation for enhancing events was developed following a user-centric approach. We describe the process of running an experiment using our custom physiological sensor platform, gathering requirements for the visualisation and finally implementing said visualisation. The end result being a collaborative artwork to enhance people's immersion into cultural events.
\end{abstract}

\section{CCS CONCEPTS}

- Human-centered computing $\rightarrow$ Information visualization;

\section{KEYWORDS}

Sensors, cultural experiences, GSR, shared experiences, interactive art, data visualisation

\section{INTRODUCTION}

A long-standing goal of our research efforts has been understanding user experience in situ during live cultural events. In particular, one of our goals is it to bring physiological sensor technology to real-world settings, making it more accessible to end-users and employ it to enhance cultural experiences. For this, we developed an easy to deploy wireless system for collecting Galvanic Skin Response data from a group of people attending a live event and visualising the data [4]. We make use of GSR sensors because we found them to be fairly unobtrusive and they have been shown to be an adequate proxy for user engagement [3]. More specifically, this paper describes a live deployment of said system at a Jazz concert, where the level of engagement of 40 guests was measured using GSR

Permission to make digital or hard copies of part or all of this work for personal or classroom use is granted without fee provided that copies are not made or distributed for profit or commercial advantage and that copies bear this notice and the full citation on the first page. Copyrights for third-party components of this work must be honored.

For all other uses, contact the owner/author(s).

MM '17, October 23-27, 2017, Mountain View, CA, USA

(C) 2017 Copyright held by the owner/author(s).

ACM ISBN 978-1-4503-4906-2/17/10.

https://doi.org/10.1145/3123266.3127919
Pablo Cesar

Centrum Wiskunde \& Informatica, Amsterdam

Delft University of Technology, Delft

The Netherlands

cesar@cwi.nl

sensors. The event was also recorded on video for post-performance analysis.

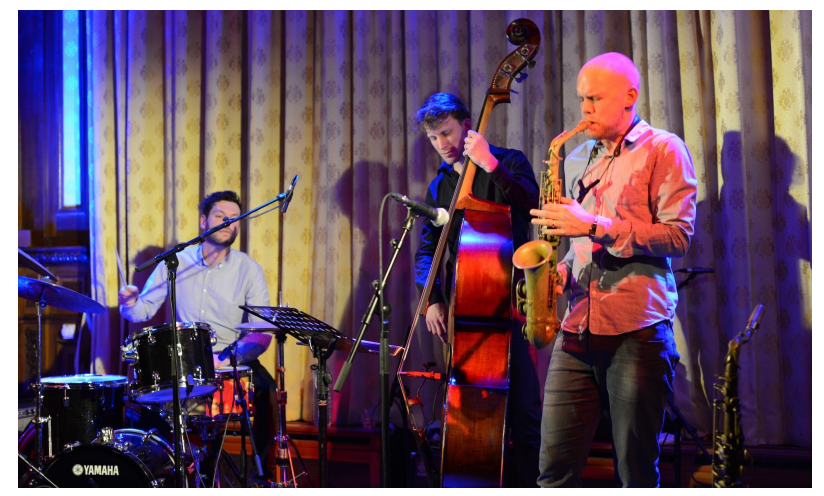

Figure 1: Jazz concert at the Goethe-Institut Amsterdam, during which the data was collected

These recordings alongside the processed sensor data and a set of requirements gathered from professionals and audience members were used to create the visualisation. Based on this process, the resulting artifact was a real-time visualisation which combines the amplitude and the beat of the music with the recorded level of engagement in the audience. The overarching goal was it to create a unique kind of collective artwork deployable at live events to elicit a higher sense of immersion among the audience. We see this as a first step towards a multi-layered concept for cultural events by attempting to merge interactive art installations with live music performances.

In the demo we want to present, we showcase a simplified version of the system that was used during the live event and display associated artifacts, such as posters and videos. Participants can try out the sensors and see the created visualisation in action.

\section{SETUP}

The Goethe-Institut is an organisation active worldwide with the goal to promote German language and culture. Their office in Amsterdam hosts monthly cultural events and among them a series of Jazz concerts in Spring 2016. We entered a collaboration to run 
an experiment which involved collecting GSR sensor data from concert attendants during one of these Jazz events. For this, we instrumented the event space at their location in the city centre of Amsterdam with two Raspberry Pis running custom-written software. Thus transforming them into receivers for the sensor data packets from our wireless GSR sensors (seen in Figure 2) by means of dedicated USB peripherals for receiving data using the RF12 protocol ${ }^{1}$. The Pis were connected via to a central machine responsible for collecting and preprocessing the received data via a wired network. The complete setup of the system can be seen in Figure 3.

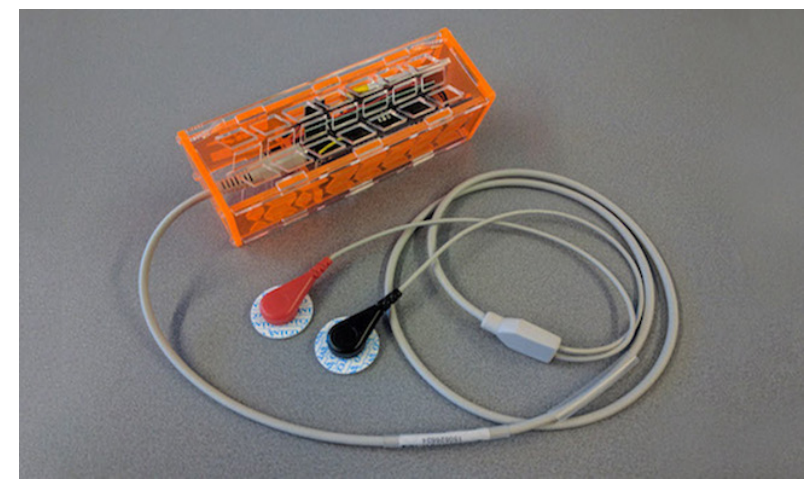

Figure 2: Custom-built GSR sensor in laser-cut casing that was handed to each participant

After the concert, the collected data was run through an algorithm to rid it of artifacts introduced - for instance - by the electrodes temporarily losing contact to a participant's skin, or data packets getting lost in transit to the receivers. As a first step, the data streams for each sensor were then aligned to the general timeline with a granularity of 1 second. As a consequence, if the receiver had received more than one GSR sample from a specific sensor within one second, it would take the average value of those samples. On the other hand, if the receiver did not receive any data from a sensor during a specific second, the value was interpolated from the two adjacent values in the timeline. Finally, the resulting file was analyzed for artifacts. These artifacts manifest themselves through sudden vertical jumps in a sensor's GSR value from one second to the next. This was fixed by differentiating the data for each sensor and zeroing all entries for which the derivative was larger than a certain value. The data was then integrated numerically using a cumulative sum to restore the original signal without the artifacts. A similar process to remove these kind of artifacts was followed by other researchers in their experiments with physiological sensor data [2].

In order to collect meaningful input for developing the visualisations, we consulted with the concert organisers, the musicians and selected audience members and asked them what kind of visuals they would find useful to enhance the concert experience and increase the audience's degree of immersion. As a definition for the term immersion we relied on one given in previous research [1]. These interviews were formalised into a set of requirements. Based on which the visualisation was implemented using Processing, a

\footnotetext{
${ }^{1}$ http://jeelabs.org/2011/06/09/rf12-packet-format-and-design/
}

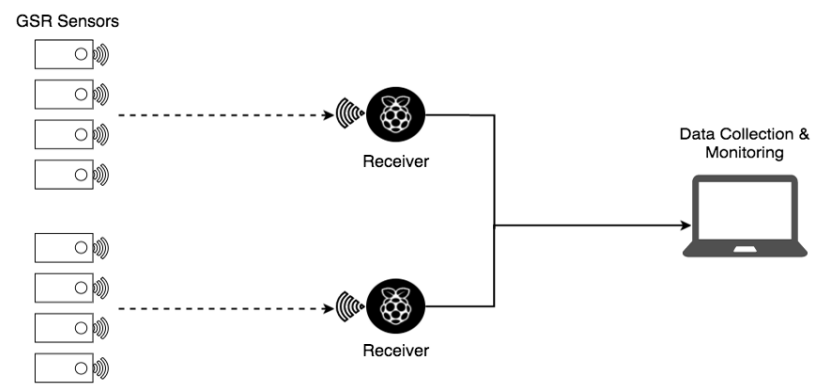

Figure 3: Architecture of the deployed system. The GSR sensors communicate with the base stations, which were connected to a central hub for processing and storing the data.

programming language that allows for the creation of visualizations based on different input streams. The implemented visualisation seen in Figure 4 takes two parameters as input: the music and the data gathered from the sensors.

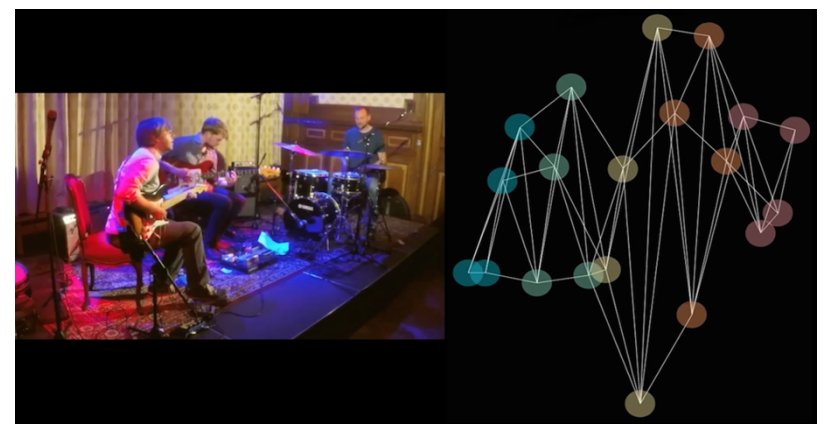

Figure 4: Visualisation taking GSR sensor data and music amplitude and beat as input.

The visualisation shows each sensor individually as a circle that moves along the y-axis of the screen according to the value of engagement as gathered by the GSR sensor. The radius of the circle responds to the amplitude of the music. Additionally, the circles were plotted in different colours, to make them easier to distinguish. Eventually, this resulted in an abstract, constantly changing, collective artwork, triggered by the feelings of the audience and the music. Even though the general mood of the audience was not explicitly shown in the visualisation, the constant movement and re-formation of the abstract visualisation could be understood as the atmosphere of the room.

\section{REFERENCES}

[1] C Jennett, A. L. Cox, P. Cairns, S. Dhoparee, A. Epps, T. Tijs, and A. Walton. 2008. Measuring and defining the experience of immersion in games. International journal of human-computer studies 66, 9 (2008), 641-661.

[2] R. Kocielnik, N. Sidorova, F. M. Maggi, M. Ouwerkerk, and J. HDM Westerink. 2013. Smart technologies for long-term stress monitoring at work. In ComputerBased Medical Systems, 2013 IEEE 26th International Symposium on. IEEE, 53-58.

[3] Peter J Lang. 1995. The emotion probe: studies of motivation and attention. American psychologist 50, 5 (1995), 372.

[4] T. Röggla, C. Wang, L. Perez Romero, J. Jansen, and P. Cesar. 2017. Tangible Air: An Interactive Installation for Visualising Audience Engagement. In Proceedings of the 2017 ACM SIGCHI Conference on Creativity and Cognition (C\&C '17). ACM, New York, NY, USA, 3. 